\title{
Technical Note: A Landmark-Based Approach to the Study of the Ear Ossicles Using Ultra-High-Resolution X-Ray Computed Tomography Data
}

\author{
Jodi L. Schmidt, ${ }^{1}$ Theodore M. Cole III, ${ }^{2}$ and Mary T. Silcox ${ }^{3 *}$ \\ ${ }^{1}$ Department of Anthropology, Trent University, Peterborough, ON, Canada K9J $7 B 8$ \\ ${ }^{2}$ Department of Basic Medical Science, School of Medicine, University of Missouri-Kansas City, Kansas City, MO 64108 \\ ${ }^{3}$ Department of Social Sciences, University of Toronto Scarborough, Toronto, ON, Canada M1C 1A4
}

KEYWORDS geometric morphometrics; error analysis; landmarks; Loris; Callicebus

\begin{abstract}
Previous study of the ear ossicles in Primates has demonstrated that they vary on both functional and phylogenetic bases. Such studies have generally employed two-dimensional linear measurements rather than three-dimensional data. The availability of Ultra- high-resolution X-ray computed tomography (UhrCT) has made it possible to accurately image the ossicles so that broadly accepted methodologies for acquiring and studying morphometric data can be applied. Using UhrCT data also allows for the ossicular chain to be studied in anatomical position, so that it is possible to consider the spatial and size relationships of all three bones. One issue impeding the morphometric study of the ear ossicles is a lack of broadly recognized landmarks. Distinguishing landmarks on the ossicles is difficult in
\end{abstract}

One of the characteristic features of definitive mammals is the presence of three ear ossicles in the middle ear cavity: the malleus, incus, and stapes (Rosowski, 1992). The malleus is the largest and most external of the ossicles and articulates with the intermediate ossicle, the incus. The third bone in the ossicular chain is the stapes; in eutherian mammals, this bone is generally the smallest and most medial of the three. The stapes articulates with the incus and its footplate is located in the oval window. The three bones of the middle ear form a functional unit. The joints between the ossicles are relatively firm, but allow for the involuntary, constant motion necessary for their function (Masali and Cremasco, 2006). These bones play a crucial role in increasing auditory receptivity by amplifying sound and overcoming the impedance mismatch between air and the fluids in the inner ear (Zwislocki, 1965; Coleman and Ross, 2004). The traditionally accepted model for how the ossicles function is that the manubrium of the malleus and the long crus of the incus serve as lever arms in a functional chain, transmitting sound vibrations from the ear drum to the footplate of the oval window. Differences in the relative lengths of these two lever arms contribute to differences in auditory perception between species (Masali et al., 1992).

Previous studies (e.g., Masali, 1971; Siori and Masali, 1983; Masali et al., 1992; Coleman and Ross, 2004; Quam and Rak, 2008) have shown that the proportions of the ear ossicles relate directly to variables critical to an animal's hearing performance and reflect taxonomic differences. There are several aspects of ear ossicle mor- part because there are only two areas of articulation in the ossicular chain, one of which (the malleus/incus articulation) has a complex three-dimensional form. A measurement error study is presented demonstrating that a suite of 16 landmarks can be precisely located on reconstructions of the ossicles from UhrCT data. Estimates of measurement error showed that most landmarks were highly replicable, with an average CV for associated interlandmark distances of less than $3 \%$. The positions of these landmarks are chosen to reflect not only the overall shape of the bones in the chain and their relative positions, but also functional parameters. This study should provide a basis for further examination of the smallest bones in the body in three dimensions. Am J Phys Anthropol 145:665-671, 2011. @ 2011 Wiley-Liss, Inc. phology that can be utilized in quantitative formulae to describe their functional significance both for individual species and for comparative purposes, including the area of the stapedial footplate and measurements of the incudal and malleolar lever arms (Coleman and Ross, 2004).

Several studies have incorporated measurements of these and other aspects of the ossicular chain (Masali, 1964, 1971; Siori and Masali, 1983; Masali et al., 1992; Coleman and Ross, 2004; Quam and Rak, 2008, Flohr et al., 2010). These studies have generally used photographs for obtaining measurements of these tiny bones and utilized a series of axes for each of the ear ossicles, with measurements being taken either parallel or per-

\footnotetext{
Additional Supporting Information may be found in the online version of this article.

Grant sponsor: NSF; Grant number: BCS-0003920; Grant sponsor: NSERC, Discovery Grants Program-Individual; Grant sponsor: University of Winnipeg, Major Grant.

*Correspondence to: Dr. Mary T. Silcox, Department of Social Sciences, University of Toronto Scarborough, 1265 Military Trail, Scarborough, Ontario, Canada M1C 1A4.

E-mail: msilcox@utsc.utoronto.ca
}

Received 6 October 2010; accepted 30 March 2011

DOI 10.1002/ajpa.21543

Published online 5 July 2011 in Wiley Online Library (wileyonlinelibrary.com). 
pendicular to these axes (e.g., Masali, 1964; Masali et al., 1992; Coleman and Ross, 2004; Quam and Rak, 2008, Flohr et al., 2010). Photographic methods for measuring the ossicles have proven challenging due to the bones' small size and the difficulty of consistently orienting them for photography. For example, Flohr et al. (2010) demonstrated that variation in photographic technique, particularly in how the ossicles were positioned, contributed significantly to the error for some measurements. The photographic method also suffers from trying to represent complicated three-dimensional structures in only two dimensions. In particular, Coleman and Ross's (2004) method of estimating the length of the lever arms rests on delineating the axis of rotation, which is represented by a line drawn from the short process of the incus to the long process of the malleus. Their method relies upon the ability to orient the ossicles so that the axis of rotation and both of the lever arms are perfectly parallel to the lens of the camera. Depending on the morphology of the ossicular chain, this orientation may not be possible for all species. Also, with the exception of Coleman and Ross's (2004) approach, axes are usually defined on the basis of convenience rather than function, making the larger significance of the measurements taken with respect to them somewhat questionable. All previous approaches to taking measurements from ossicles have dealt with them either in isolation (Masali, 1964, 1971; Siori and Masali, 1983; Masali et al., 1992; Quam and Rak, 2008, Flohr et al., 2010), or with only the incus and malleus in articulation (Coleman and Ross, 2004), so that spatial relationships between the three ossicles are lost. For specimens in which the ossicles are still in situ, using UhrCT data preserves these relationships, which may allow for an enhanced perspective on the relationship between the structure and function of these tiny bones. Since the ear drum remains in place in many macerated specimens and the stapes is typically still articulated with the oval window, the ossicular chain is often preserved in its original location and orientation in skeletonized specimens. The approach described in this paper could also be applied to UhrCT data from specimens in which the soft tissues are still intact.

An alternative approach to characterizing the morphology and function of the ear ossicles is to make use of landmarks that can be defined based on anatomy and function, rather than on a relationship to a set of arbitrarily defined axes. Anatomical landmarks are unambiguously defined, biologically meaningful loci that are able to be located repeatedly with a high degree of precision (Richtsmeier et al., 1995). No one has ever proposed a set of standardized anatomical landmarks for the ear ossicles, likely due to their small size, the lack of easily characterized sutures within the ossicular chain, and the complicated shapes of the ossicles themselves. These factors make the definition of traditional single-point landmarks impossible on the ossicular chain. An alternative approach is to utilize "fuzzy landmarks". Valeri et al. (1998:114) describe fuzzy landmarks as representing “. . . a biological structure that is precisely delineated and that corresponds to a locus of some biological significance, but occupies an area that is larger than a single point." This type of landmark is most easily placed in a 3D environment. Fuzzy landmarks can make it possible to capture aspects of anatomical structures that are typically difficult to quantify because of their complicated morphology, or that lack landmarks that fit the tradi- tional definition. However, the nature of fuzzy landmarks makes it particularly important to be able to verify their accuracy. Each fuzzy landmark holds more than a single point location, which may make them more difficult to unambiguously locate than traditional landmarks. For this reason, a measurement error study is essential to demonstrate that they can be precisely located (sensu Richtsmeier et al., 1995) before they can be considered reliable.

In this paper, we define a suite of 16 landmarks that can be used to describe the ossicular chain both functionally and morphologically (Table 1). Because of the complicated nature of the ear ossicles and their articulations with each other, the majority of these landmarks are fuzzy landmarks. By utilizing fuzzy landmarks and placing them in a $3 \mathrm{D}$ environment, we avoid the issues identified as significant by Flohr et al. (2010) with respect to the photographic technique. We demonstrate that fuzzy landmarks of the ossicular chain can be used to produce both functionally relevant and precise results.

\section{MATERIALS AND METHODS}

In standard medical CT, a specimen can generally be sampled, at best, every $1 \mathrm{~mm}$ along its length (i.e., the slice thickness is $1 \mathrm{~mm}$ ). For small mammals, the ear ossicles are typically only $1-4 \mathrm{~mm}$ in their largest dimension, meaning that each bone would be captured in at most four slices using medical CT, which would make accurate three-dimensional reconstruction impossible. However, Ultra high-resolution X-ray computed tomography (UhrCT) allows for much thinner slices. For this study, skulls of Loris tardigradus (University of Massachusetts [UM] APC70) and Callicebus torquatus (Field Museum of Natural History [FMNH] 70695) were utilized in which the ear ossicles are preserved in situ. These datasets, with slice thicknesses more than 25 times thinner than that used in conventional medical $\mathrm{CT}$, sample the ear ossicles in over 80 slices, allowing for a very accurate reconstruction.

\section{Scanning protocol}

The specimens studied here were embedded in cylinders of floral foam, placed in plastic vials, and mounted on the OMNI-X Industrial Scanner at the Center for Quantitative Imaging at Pennsylvania State University. The scans were taken in volume mode, with 21 slices per volume. Each rotation included 2,400 views covering $360^{\circ}$. Slices were reconstructed using all 2,400 views to produce $1024 \times 1024$-pixel images that were stored as 16-bit TIFF files. The resulting slice thickness for the Loris tardigradus specimen is $37.03 \mu \mathrm{m}$ and the pixel size is $34.28 \mu \mathrm{m} \times 34.28 \mu \mathrm{m}$. For the Callicebus torquatus specimen slice thickness is $41.44 \mu \mathrm{m}$ and the pixel size is $35.16 \mu \mathrm{m} \times 35.16 \mu \mathrm{m}$.

\section{Ear ossicle reconstruction protocol}

The datasets were initially viewed to determine whether or not the ossicles were present, and in which slices they were sampled. The datasets were then reduced in size to the smallest volume that could contain the ear ossicles by removing extra slices and cropping in cropvoi (unpublished DOS program developed by Nathan Jeffrey, University of Liverpool). The data were read into ImageJ (Rasband, 1997-2009), and all extraneous data (e.g., nonossicle bone, ligaments) were removed from 


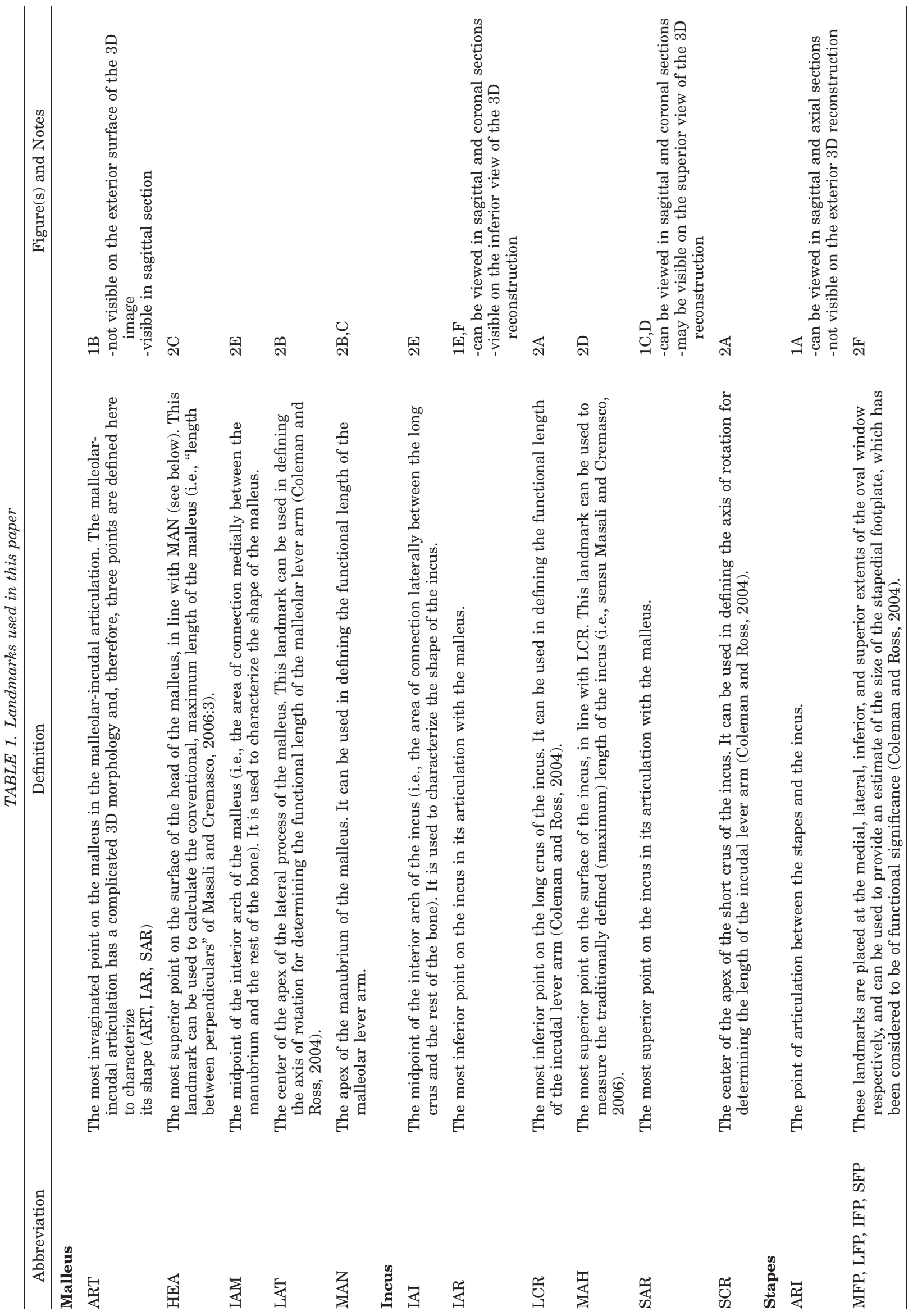



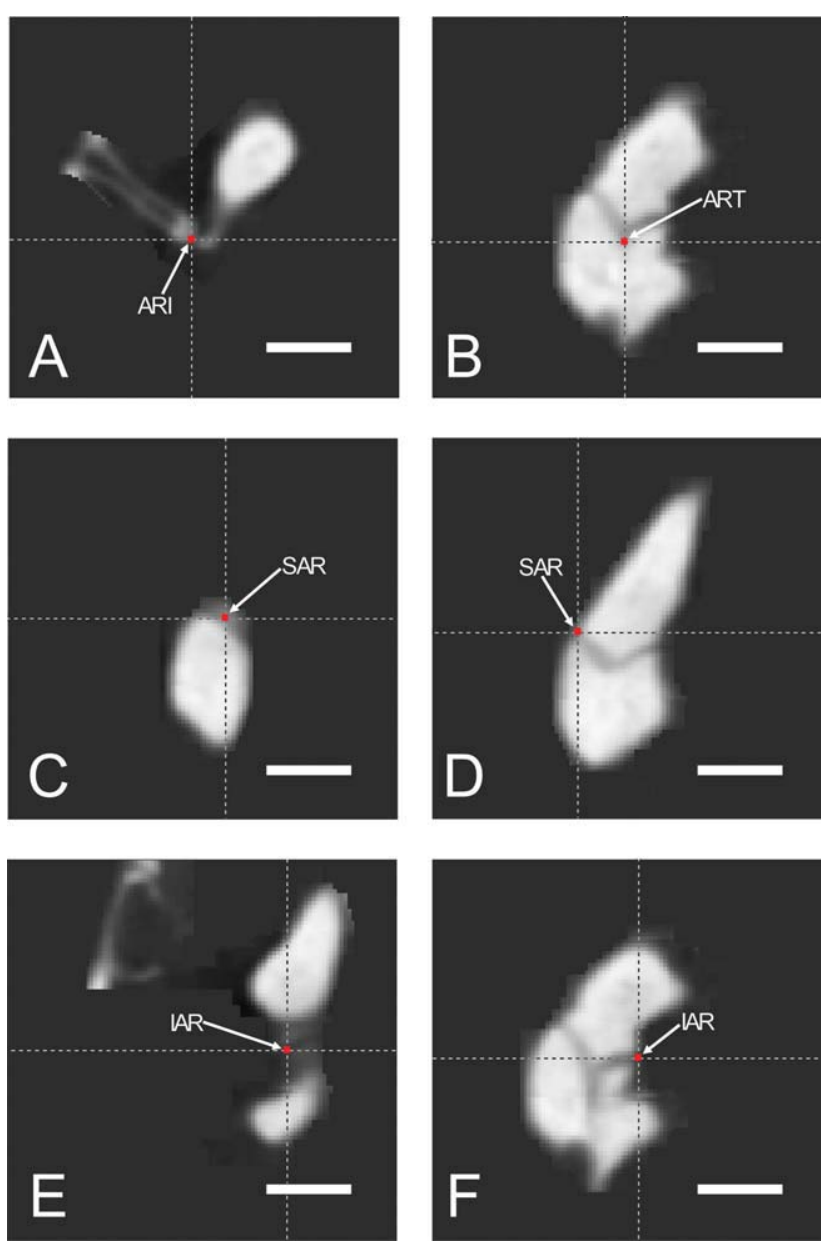

Fig. 1. Two-dimensional cross-sectional images of the ear ossicles of Loris tardigradus ([UM] APC70) created in etdips (http://www.cc.nih.gov/cip/software/etdips/) showing the complicated interior morphology of the ossicular articulations and the landmarks used to define these areas. Scale bars $=1 \mathrm{~mm}$. A: ARI is located at the point of articulation between the stapes and the incus. This image is in axial view. B-F: SAR, ART, and IAR serve to characterize the complex malleolar-incudal articulation. SAR and ART can only be placed on the cross-sectional images, in either the sagittal or coronal views. B, D, F: sagittal views; C, E: coronal views.

each slice. Alternative, automated methods of isolating the ear ossicles have proven less successful because these tiny bones are often very close to, and in some cases touching, surrounding tissues. This means that such methods are unsuccessful at consistently isolating all of the relevant anatomy. The cropped and trimmed dataset was then read into etdips (http://www.cc.nih.gov/ cip/software/etdips/) to produce a three-dimensional surface reconstruction of the bones (threshold values for Loris = 2034-8217; Callicebus $=3,600-8,386)$ (Figs. 1 and 2).

\section{Measurement error analysis}

Two of the authors (JLS \& MTS) independently performed 20 landmark-placement trials each on the same Loris tardigradus specimen ([UM] APC70). Similarly, they performed 10 trials each on the same Callicebus torquatus specimen ([FMNH] 70695). For each specimen,
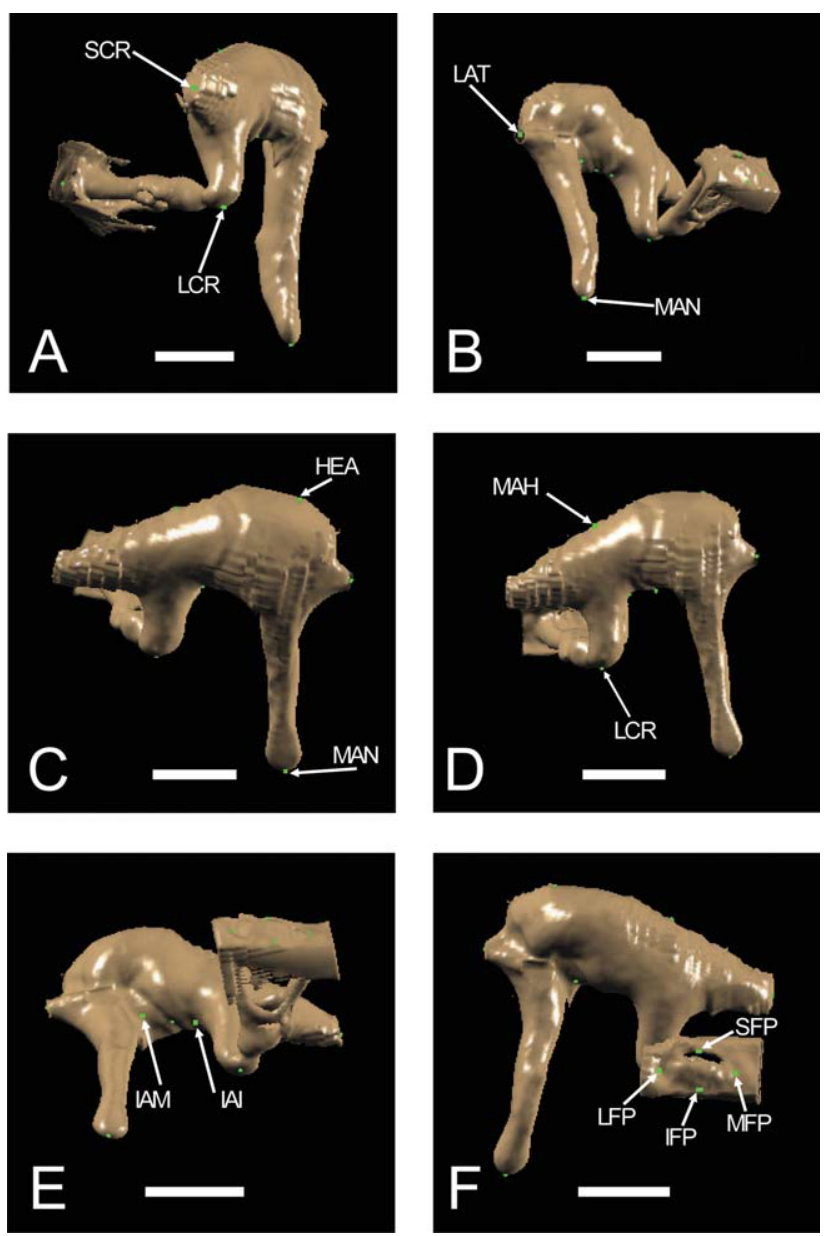

Fig. 2. Three-dimensional reconstructions of the ear ossicles of Loris tardigradus (UM) APC70 created in etdips (http:// www.cc.nih.gov/cip/software/etdips/) showing landmarks used to define both functional and morphological features of the malleus, incus and stapes. A, B: The landmarks illustrated in these images are particularly important functionally. LAT and SCR together define the axis of rotation, from which the functional lengths of the malleus and incus can be measured. These measurements are made from the axis of rotation to MAN and LCR respectively (Coleman and Ross, 2004). C, D: These four landmarks define important functional and morphological features of the malleus and incus. MAH and LCR can be used to calculate the length of the incus sensu Masali and Cremasco, 2006. HEA and MAN can be used to calculate the conventional length of the malleus ("length between perpendiculars" of Masali and Cremasco, 2006: 3). E: The two landmarks illustrated in this figure, IAM and IAI, are located in the interior arches of the malleus and incus respectively, and serve to help characterize these bones morphologically. F: This figure shows the four landmarks, MFP, LFP, IFP, and SFP, which define the parameters of the oval window. These landmarks are important for characterizing both the morphology of this region and in providing an estimate of the size of the oval window and thus the stapedial footplate.

the same reconstruction, based on the same threshold values, was used for all trials. Individual trials were separated by at least eight hours. Data were collected from objects in a fixed coordinate system (that is, the threedimensional coordinate system defined by the scanner). This means that measurement error estimation is very straightforward since it is not necessary to use any type of fitting criteria (e.g., Procrustes algorithms) to make comparisons between trials (see discussion in Richtsmeier 
TABLE 2. "Overall" standard deviations (Sigma) for Observers 1 and 2 and for pooled data for Loris tardigradus ([UM] APC70)

\begin{tabular}{|c|c|c|c|c|c|c|c|c|}
\hline & \multicolumn{2}{|c|}{ Observer 1 (JLS) } & \multicolumn{2}{|c|}{ Observer 2 (MTS) } & \multicolumn{4}{|c|}{ Pooled } \\
\hline & Sigma & Rank & Sigma & Rank & Sigma & Rank & $\begin{array}{l}\text { Percent } \\
\text { smallest } \\
\text { distance }\end{array}$ & $\begin{array}{l}\text { Percent } \\
\text { average } \\
\text { distance }\end{array}$ \\
\hline MAN & 0.0125 & 12 & 0.0123 & 16 & 0.0124 & 15 & $0.64 \%$ & $0.46 \%$ \\
\hline LAT & 0.0043 & 16 & 0.0161 & 15 & 0.0102 & 16 & $1.00 \%$ & $0.47 \%$ \\
\hline HEA & 0.0471 & 3 & 0.0628 & 3 & 0.0550 & 3 & $7.69 \%$ & $2.82 \%$ \\
\hline ART & 0.0124 & 13 & 0.0272 & 10 & 0.0198 & 12 & $4.30 \%$ & $1.33 \%$ \\
\hline IAM & 0.0411 & 5 & 0.0647 & 2 & 0.0529 & 4 & $12.91 \%$ & $3.59 \%$ \\
\hline LCR & 0.0244 & 8 & 0.0277 & 9 & 0.0261 & 9 & $10.72 \%$ & $1.61 \%$ \\
\hline SCR & 0.0134 & 11 & 0.0345 & 6 & 0.0240 & 10 & $1.77 \%$ & $1.10 \%$ \\
\hline IAR & 0.0717 & 1 & 0.0543 & 4 & 0.0360 & 2 & $22.23 \%$ & $4.60 \%$ \\
\hline SAR & 0.0613 & 2 & 0.0857 & 1 & 0.0737 & 1 & $12.69 \%$ & $4.49 \%$ \\
\hline MAH & 0.0425 & 4 & 0.0421 & 5 & 0.0423 & 5 & $7.29 \%$ & $2.55 \%$ \\
\hline IAI & 0.0304 & 7 & 0.0261 & 12 & 0.0282 & 6 & $9.97 \%$ & $2.12 \%$ \\
\hline ARI & 0.0367 & 6 & 0.0173 & 14 & 0.0270 & 7 & $2.63 \%$ & $1.74 \%$ \\
\hline MFP & 0.0180 & 10 & 0.0283 & 8 & 0.0232 & 11 & $4.72 \%$ & $1.14 \%$ \\
\hline LFP & 0.0219 & 9 & 0.0306 & 7 & 0.0262 & 8 & $5.27 \%$ & $1.41 \%$ \\
\hline SFP & 0.0115 & 14 & 0.0267 & 11 & 0.0191 & 13 & $4.37 \%$ & $1.05 \%$ \\
\hline IFP & 0.0110 & 15 & 0.0235 & 13 & 0.0172 & 14 & $3.95 \%$ & $0.89 \%$ \\
\hline
\end{tabular}

The two rightmost columns indicate the relative amounts of error for the pooled data, relative to the smallest distance for which a landmark is an endpoint and the average distance. The values in boldface denote landmarks where either relative-error measure exceeds $10 \%$.

The ranks indicate which landmarks are most error-prone (low ranks) versus least error-prone (high ranks).

et al., 1995). This approach also allows for the calculation of separate error along each of the three dimensions (see Supporting Information). Detailed descriptions of the calculations are provided by Valeri et al. (1998: Appendix).

Because of the fixed coordinate system, landmark-specific, coordinate-wise ( $x$-, $y$-, and $z$-axis) means, variances, and standard deviations can be computed directly from the data, as one would with other continuous measurements (e.g., Sokal and Rohlf, 1995; Supporting Information Tables 1 and 2). Valeri et al. (1998) describe how to perform equivalent computations using matrix algebra. In addition to describing the measurement error for each landmark in a coordinate-wise fashion, we may also quantify a landmark's error in a more "overall" sense, considering error in all three coordinate axes simultaneously. This approach requires matrix algebra (Valeri et al., 1998). Suppose we collect three-dimensional landmark data from an object with $K$ landmarks in $n$ trials. The three-dimensional data for Trial $i$ can be stored in a $K \times 3$ matrix called $\mathbf{T}_{i}$, where each row consists of the $x$-, $y$-, and $z$-axis data for a landmark. The mean coordinates of all $n$ trials are placed in a $K \times 3$ matrix called $\hat{\mathbf{M}}$. Let us mean-center each trial's data by subtracting the mean matrix from the trial's raw measurement data: $\hat{\mathbf{T}}_{i}^{c}=\mathbf{T}_{i}-\hat{\mathbf{M}}$, where the $c$ superscript denotes "centered." We can now compute a $K \times K$ matrix called $\hat{\Sigma}_{K}$ :

$$
\hat{\mathbf{\Sigma}}_{K}=\frac{1}{3 n} \Sigma\left\{\left(\hat{\mathbf{T}}_{i}^{c}\right)\left(\hat{\mathbf{T}}_{i}^{c}\right)^{T}\right\}
$$

where the $T$ superscript denotes a matrix transposition. The matrix has the form:

$$
\hat{\mathbf{\Sigma}}_{K}=\left[\begin{array}{cccc}
\sigma_{1}^{2} & 0 & 0 & 0 \\
0 & \sigma_{2}^{2} & 0 & 0 \\
0 & 0 & \ddots & 0 \\
0 & 0 & 0 & \sigma_{K}^{2}
\end{array}\right]
$$

Each of the diagonal elements is the "overall" variance of the measurement error for the corresponding landmark.
We can convert each of the variances to standard deviations by taking their square roots. The off-diagonal elements are all zeros, indicating that there are no correlations in measurement errors across different landmarks.

In practical applications, it may be difficult to interpret the errors in individual landmarks without placing them into some kind of broader context. For example, we may not be able to tell by simple inspection how large the variance in a landmark must be before it is considered "too large", leading us to conclude that the landmark was too error-prone to be used reliably. To put the estimates of overall error into perspective, a type of coefficient of variation was used, with the aim of seeing how errors in landmarks contribute to errors in the interlandmark distances they define (e.g., Ross and Williams, 2008). We take a very conservative "worst-case" scenario (percent smallest distance) to see how landmark errors affect the smallest interlandmark distances they define (where their impacts will be relatively largest). Each landmark's standard deviation (either coordinate-wise or overall) is divided by the mean of the smallest distance for which that landmark is an endpoint. We also computed percent average distance to get a less conservative measure of each landmark's relative error (Tables 2 and 3).

\section{RESULTS}

We calculated the measurement errors separately for both observers, giving us two measures of intraobserver error. We also pooled the observers' data together to get some measure of interobserver error. Pooling the data was possible because we used a common, instrumentbased coordinate system. Supporting Information Tables 1 and 2 show the axis-specific standard deviations for each landmark, both for each observer and for the pooled data. Overall, the highest standard deviation values are distributed almost evenly amongst the three axes.

Tables 2 and 3 show the "overall" measurement error for each landmark, again for each observer and for the pooled data. The standard deviations are ranked from most error-prone (\#1) to least error-prone (\#16). For both 
TABLE 3. "Overall" standard deviations (Sigma) for Observers 1 and 2 and for pooled data for Callicebus torquatus (FMNH 70695)

\begin{tabular}{|c|c|c|c|c|c|c|c|c|}
\hline & \multicolumn{2}{|c|}{ Observer 1 (JLS) } & \multicolumn{2}{|c|}{ Observer 2 (MTS) } & \multicolumn{4}{|c|}{ Pooled } \\
\hline & Sigma & Rank & Sigma & Rank & Sigma & Rank & $\begin{array}{l}\text { Percent } \\
\text { smallest } \\
\text { distance }\end{array}$ & $\begin{array}{l}\text { Percent } \\
\text { average } \\
\text { distance }\end{array}$ \\
\hline MAN & 0.0152 & 12 & 0.0147 & 16 & 0.0150 & 16 & $1.04 \%$ & $0.52 \%$ \\
\hline LAT & 0.0131 & 16 & 0.0263 & 9 & 0.0197 & 13 & $2.07 \%$ & $0.75 \%$ \\
\hline HEA & 0.0535 & 2 & 0.0471 & 4 & 0.0503 & 3 & $7.82 \%$ & $1.95 \%$ \\
\hline $\mathrm{ART}$ & 0.0261 & 6 & 0.0340 & 7 & 0.0301 & 6 & $8.38 \%$ & $1.38 \%$ \\
\hline IAM & 0.0272 & 5 & 0.0418 & 5 & 0.0345 & 5 & $4.15 \%$ & $1.69 \%$ \\
\hline LCR & 0.0159 & 11 & 0.0148 & 15 & 0.0154 & 14 & $5.74 \%$ & $0.69 \%$ \\
\hline SCR & 0.0147 & 14 & 0.0156 & 14 & 0.0152 & 15 & $0.75 \%$ & $0.51 \%$ \\
\hline IAR & 0.0502 & 3 & 0.0766 & 1 & 0.0634 & 2 & $17.5 \%$ & $3.44 \%$ \\
\hline SAR & 0.0198 & 10 & 0.0342 & 6 & 0.0270 & 7 & $7.53 \%$ & $1.16 \%$ \\
\hline MAH & 0.0792 & 1 & 0.0501 & 3 & 0.0646 & 1 & $6.32 \%$ & $2.64 \%$ \\
\hline IAI & 0.0232 & 8 & 0.0562 & 2 & 0.0397 & 4 & $10.97 \%$ & $2.12 \%$ \\
\hline ARI & 0.0148 & 13 & 0.0255 & $1 \overline{1}$ & 0.0202 & 12 & $2.11 \%$ & $0.93 \%$ \\
\hline MFP & 0.0228 & 9 & 0.0233 & 12 & 0.0230 & 10 & $3.26 \%$ & $0.81 \%$ \\
\hline LFP & 0.0134 & 15 & 0.0285 & 8 & 0.0210 & 11 & $2.87 \%$ & $0.81 \%$ \\
\hline SFP & 0.0238 & 7 & 0.0256 & 10 & 0.0247 & 8 & $3.87 \%$ & $0.99 \%$ \\
\hline IFP & 0.0296 & 4 & 0.0176 & 13 & 0.0236 & 9 & $3.71 \%$ & $0.88 \%$ \\
\hline
\end{tabular}

The ranks indicate which landmarks are most error-prone (low ranks) versus least error-prone (high ranks). The two rightmost columns indicate the relative amounts of error for the pooled data, relative to the smallest distance for which a landmark is an endpoint and the average distance. The values in boldface denote landmarks where either relative-error measure exceeds $10 \%$.

observers, the three most error-prone landmarks were IAR, HEA, and MAH. LAT was the least error-prone in the pooled results for the Loris, while MAN was the least error prone for the Callicebus.

When the pooled standard-deviation estimates were used to compute "coefficients of variation" for the smallest distances to which they belong, relative errors were very high (>10\%) for IAM, LCR, IAR, and SAR, for Loris and IAR and IAI for Callicebus, indicating that these landmarks are poor choices for defining very small distances. However, when expressed as a percentage of average distances, all of the landmarks were below $5 \%$ and most were below $3 \%$, indicating a high degree of precision in general.

\section{DISCUSSION}

The results of the measurement error study demonstrate that the 16 landmarks defined here could generally be located reliably, although a few landmarks performed poorly when it came to precisely defining small interlandmark distances. When working with very small bones like ear ossicles, it is impossible to avoid measuring very small distances; however, not all of the interlandmark distances calculated in this study are equally significant in a biological sense. We consider the interlandmark distances that define functional lengths to be of greater potential significance for characterizing intrataxonomic variation than distances that serve to simply characterize the morphology of the ossicular chain. Functional parameters are potentially subject to more constraints than parameters that are not clearly tied to function, suggesting that they may be less subject to change. With the exception of LCR, the landmarks possibly tied to functional parameters are among the least error-prone. While LCR performed poorly in the percentsmallest-distance analysis for the Loris, its performance in the percent-average-distance analysis is much more acceptable. In general, the landmarks that performed the poorest in terms of percent smallest distance are located very close to other landmarks (e.g., LCR and ARI; IAR and IAI), so that any deviation in their placement would greatly affect the extremely small distances between these landmarks and their near neighbors.
While our landmarks that participate in functional lengths performed best, the majority of the landmarks, including those important for defining the overall morphology of the ossicular chain, still fell within the traditionally accepted error range (i.e., $<5 \%$ ). In fact, while landmarks along known sutures have typically been found to be more easily replicable, the ranking of our 16 landmarks shows that with accurate three-dimensional images, "fuzzy" landmarks can outperform those situated on a suture or articular surface.

In this study, many of the problems associated with defining and precisely locating landmarks on these small bones have been overcome. These results provide a basis for future studies quantifying both functional and morphological aspects of the ear ossicles.

\section{ACKNOWLEDGMENTS}

The UhrCT scanning was performed by Özgen Karacan and Tim Ryan (Center for Quantitative Imaging, Penn State University). Thanks to Y. Carter, M. Flook, A. Grader, P. Halleck, N. Jeffrey, Ö. Karacan, T. Ryan, F.S. Spoor, A. Walker, and Y. Zelege.

\section{LITERATURE CITED}

Coleman MN, Ross CF. 2004. Primate auditory diversity and its influence on hearing performance. Anat Rec A 281:1123-1137.

Flohr S, Leckelt J, Kierdorf U, Kierdorf H. 2010. How reproducibly can human ear ossicles be measured? A study of interobserver error. Anat Rec 293:2094-2106.

Masali M. 1964. Dati sulla variabilita morphometrica e ponderale degli ossicini dell'udito nell'Uomo. Archivio Italiano di Anatomia ed Embriologia 69:435-446.

Masali M. 1971. Morphometry of ear bones of some New World Primates. In: Biegert J, Leutenegger W, editors. Taxonomy, anatomy, reproduction. Proceedings of the 3rd International Congress of Primatology, 1970, Vol. 1. Zurich: Karger, Basel. p 226-232.

Masali M, Borgognini Tarli S, Maffei M. 1992. Auditory ossicles and the evolution of the primate ear: a biomechanical approach. In: Wind J, editor. Language origin: a multidisciplinary approach. Netherlands: Kluwer. p 67-86. 
Masali M, Cremasco MM. 2006. Hoc alterum auditus organi ossiculum est: ear ossicles in physical anthropology. Hum Evol 21:1-17.

Quam R, Rak Y. 2008. Auditory ossicles from southwest Asian Mousterian sites. J Hum Evol 54:414-433.

Rasband WS. 1997-2009. ImageJ. Software and documentation. U. S. National Institutes of Health, Bethesda, Maryland, USA, Available at: http://rsb.info.nih.gov/ij/.

Richtsmeier JT, Paik CH, Elfert PC, Cole III TM, Dahlman HR. 1995. Precision, repeatability and validation of the localization of cranial landmarks using computed tomography scans. Cleft Palate Craniofac J 32:217-227.

Rosowski JJ. 1992. Hearing in transitional mammals: predictions from the middle-ear anatomy and hearing capabilities of extant mammals. In: Webster DB, Ray RR, Popper AN, editors. The evolutionary biology of hearing. New York: Springer-Verlag. p 615-631.
Ross AH, Williams S. 2008. Testing repeatability and error of coordinate landmark data acquired from crania. J Forensic Sci 53:782-785.

Siori MS, Masali M. 1983. Multivariate analysis of the ear bones of primates in taxonomic and evolutionary surveys. J Hum Evol 12:563-571.

Sokal RR, Rohlf FJ. 1995. Biometry: the principles and practice of statistics in biological research, 3rd ed. New York: W.H. Freeman and Co.

Valeri CJ, Cole III TM, Lele S, Richtsmeier JT. 1998. Capturing data from three-dimensional surfaces using fuzzy landmarks. Am J Phys Anthropol 107:113-124.

Zwislocki J. 1965. Analysis of some auditory characteristics. In Luce RD, Bush RR, Galanter E, editors. Handbook of mathematical psychology. New York: Wiley. p 1-98. 